\title{
Marcadores de fragilidade física preditivos de sintomas depressivos em pessoas idosas da atenção primária à saúde
}

\author{
Predictive physical frailty markers of depressive symptoms in older people in primary health care
}

\section{Resumo}

Objetivo: identificar quais os marcadores de fragilidade física predizem os sintomas depressivos (SD) em pessoas idosas assistidas na Atenção Primária à Saúde. Método: estudo quantitativo de corte transversal e correlacional desenvolvido em uma Unidade Básica de Saúde em Curitiba, (PR), Brasil, com amostra de 389 pessoas idosas. Coletaram-se os dados de janeiro a outubro de 2019, por meio de questionário sociodemográfico e clínico, escala de depressão (Center for Epidemiological Studies) e testes que compõem o fenótipo da fragilidade física. Para as análises, utilizou-se estatística descritiva, inferencial (quiquadrado de Pearson), nível de significância de $p \leq 0,05)$, e regressão logística reportado a estimativa, valor $p$ (teste de Wald). Razão de Prevalência com intervalo de confiança $95 \%$. Resultados: das 389 pessoas idosas, 103 (26,5\%) apresentaram SD; entre eles 63 (61,2\%) eram pré-frágeis, $19(18,4 \%)$ frágeis e $21(20,4 \%)$ não frágeis. Associaram-se aos SD os marcadores fadiga/exaustão $(p \leq 0,001)$, redução do nível de atividade física $(p \leq 0,001)$, perda de peso não intencional $(p=0,003)$ e a condição de pré-fragilidade e fragilidade $(p \leq 0,001)$. O modelo preditivo para os SD incluiu os marcadores fadiga/exaustão (RP: 5,12; IC95\%; 3,81-6,87; $p<0,0001)$ e redução do nível de atividade física (RP: 2,16, IC95\%; 1,45- 3,22; $p<0,0001)$. Conclusão: os marcadores do fenótipo fadiga/exaustão e redução da atividade física são preditores dos SD em pessoas idosas. Esse resultado ressalta a importância e a necessidade da avaliação desses marcadores e da efetividade de ações para o combate ao sedentarismo em pessoas idosas da atenção primária à saúde.

\section{Abstract}

Objective: to identify which markers of physical frailty predict depressive symptoms (DS) in old people assisted in Primary Health Care. Method: this is a quantitative, descriptive, and correlational cross-sectional study was carried out at the Basic Health Unit in Curitiba,
Palavras-chave: Idoso fragilizado. Depressão. Fragilidade. Atenção Primária à Saúde

Universidade Federal do Paraná (UFPR), Programa de pós-graduação em enfermagem (PPGENF/ UFPR), Grupo Multiprofissional de Pesquisa sobre Idosos (GMPI). Curitiba, Paraná, Brasil.

Os autores declaram não haver conflito na concepção deste trabalho.

Não houve financiamento para a execução deste trabalho. 
(PR), Brazil, with a sample of 389 old people. Data were collected from January to October 2019, using a sociodemographic and clinical questionnaire, depression scale (Center for Epidemiological Studies) and to evaluate the phenotype of Frailty. The statistical analyzes were performed using descriptive statistics, inferential statistics (Pearson's chi-square), with statistical significance level $p<0.05$, and logistic regression, reporting the estimate, $p$ value (Wald test), Prevalence Ratio with a 95\% confidence interval. Results: of the 389 old people, $103(26.5 \%)$ had DS; among these 63 (61.2\%) were pre-frail, 19 (18.4\%) frail and $21(20.4 \%)$ non-frails. The markers fatigue/exhaustion $(p \leq 0.001)$ reduced level of physical activity $(p \leq 0.001)$, unintentional weight loss $(p=0.003)$ and the condition of pre-frailty and frailty were associated with DS s ( $p=\leq 0.001)$. The final predictive model

Keywords: Frail elderly. Depression. Frailty. Primary Health Care. and reduced level of physical activity (PR 2.16, 95\%CI \%; 1.45-3.22; $p<0.0001$ ). Conclusion: the markers of fatigue/exhaustion phenotype and reduced physical activity are predictors of DS in the old people. This result highlights the importance and need to assess these markers, and the effectiveness of actions to combat sedentary lifestyle in the old people in primary health care.

\section{INTRODUÇÃO}

A fragilidade física em pessoas idosas é considerada uma síndrome geriátrica e tornou-se uma preocupação para a saúde pública, em razão do aumento da expectativa de vida da população. Essa síndrome apresenta-se como uma condição multidimensional, com comprometimento de diferentes funções biológicas. Ela também foi definida por especialistas como um "estado clínico caracterizado por um aumento da vulnerabilidade no indivíduo, quando exposto a estressores internos e externos", além de ser um dos principais contribuintes para o declínio funcional e mortalidade precoce em pessoas idosas ${ }^{1}$.

A identificação da fragilidade por meio do fenótipo físico abrange a avaliação de cinco marcadores: perda de peso não intencional, autorrelato de fadiga/ exaustão, redução do nível de atividade física, diminuição da velocidade da marcha e da força de preensão manual. O quantitativo de marcadores identificados classifica as pessoas idosas em não frágeis (nenhum), pré-frágeis (um ou dois) ou frágeis (três ou mais $)^{2}$.

A fragilidade apresenta expressiva prevalência em pessoas idosas da comunidade. Uma revisão sistemática com metanálise analisou amostra de 13.392 indivíduos ( $\geq 65$ anos) de 22 países europeus, e identificou $18 \%$ de pessoas idosas frágeis ${ }^{3}$. $\mathrm{Na}$ América do Sul, destaca-se estudo que incluiu o Brasil, Chile, Peru, Colômbia, Equador, Argentina e Venezuela, que identificou prevalência média de $21,7 \%$ de fragilidade, sendo que um a cada cinco idosos residentes na comunidade foi identificado como frágil ${ }^{4}$.

Embora o fenótipo seja físico, a multifatoriedade da fragilidade permite observar a relação com aspectos cognitivos, psicológicos e de funcionamento social ${ }^{5}$. A relação entre a fragilidade e os fatores psicossociais foi investigada em estudo com metanálise que incluiu 8.023 indivíduos. Os frágeis apresentaram chance duas vezes maior para desenvolver depressão (OR: 2.64; IC 95\%: 1,59-4,37), comparados aos nãofrágeis, e aqueles com depressão apresentaram três vezes mais chance para desenvolver fragilidade (OR:3.72; IC 95\%: 1,95-7,08), o que demonstra a relação bidirecional entre as condições ${ }^{6}$.

De forma semelhante à fragilidade, os Sintomas Depressivos (SD) também são comuns no processo de envelhecimento. Além disso, estudos mostram relacionados aos marcadores de fragilidade, pois as pessoas idosas nessa condição podem reduzir o nível de atividade física, diminuição da força e apresentar fadiga/exaustão ${ }^{7}$. Ainda, é comum a perda de interesse, o declínio da capacidade funcional e da participação social, com tendência ao isolamento ${ }^{8}$.

O impacto potencial dos SD e da fragilidade incidente em pessoas idosas foi investigado em estudo realizado nos países latino-americanos (Cuba, República Dominicana, México, Venezuela, Porto 
Rico e Peru) em uma coorte de 12.844 idosos. Os resultados demonstraram relação recíproca entre ambas as condições e risco aumentado de $59 \%$ $(\mathrm{HR}=1,59$; IC 95\%: 1,40-1,80) pessoas idosas com SD desenvolverem fragilidade?

A relação prospectiva entre o risco aumentado de fragilidade e os SD e a interação recíproca entre as condições foram demonstradas por estudos que identificaram além de sintomas somáticos, a associação entre a condição e alguns marcadores em relação aos SD. Entre eles, são frequentes, a fadiga/exaustão, a redução da velocidade na marcha, a diminuição da atividade física, a perda de peso não intencional, as comorbidades e os prejuízos cognitivos e funcionais ${ }^{10}$. Assim como os marcadores, a condição de fragilidade também foi investigada na ralação transversal e longitudinal em estudo realizado na China com 1.264 pessoas idosas. Verificaram-se associações entre a condição de pré-frágil e frágil, além do marcador força de preensão manual com a ocorrência de SD nas pessoas idosas ${ }^{11}$.

Os especialistas consideraram necessária a avaliação de sintomas apresentados pelos idosos, especialmente em relação à fadiga/exaustão e suas causas, uma vez que esse componente demonstrou ser o primeiro sintoma a se manifestar em adultos mais velhos ${ }^{1}$.

Esclarecer a relação entre fragilidade e os SD tem implicações importantes para a compreensão dos fatores que contribuem para a etiologia e prognóstico dessas variáveis. O destaque para os marcadores do fenótipo de fragilidade física é relevante, visto que, em alguns estudos, observa-se o predomínio deles, no entanto são escassos os estudos sobre como eles se comportam na predição de SD e na condição de fragilidade da pessoa idosa.

Frente ao exposto, o objetivo do estudo foi identificar quais marcadores de fragilidade física podem predizer os SD em pessoas idosas assistidas na Atenção Primária à Saúde.

\section{MÉTODO}

Trata-se de estudo do tipo transversal, correlacional, realizado em uma Unidade Básica de Saúde (UBS) que compõe a rede de Atenção Primária da cidade de Curitiba (PR), Brasil, no período de janeiro a outubro de 2019. A UBS foi eleita entre outras 110 unidades do município, por possuir uma população de 23.890 pessoas com cadastro ativo, sendo 4.439 pessoas idosas, o que representa 18,58\% da população adscrita.

Os participantes foram pessoas idosas de ambos os sexos, com idade igual ou superior a 60 anos, cadastrados e residentes na área de abrangência da UBS. A amostra não probabilística, representativa da população das pessoas idosas atendidas na UBS foi definida por cálculo amostral que indicou 354 idosos, para os quais acrescentou-se $10 \%$ pela possibilidade de perdas, a amostra final foi constituída por 389 pessoas idosas. Considerou-se o índice de confiança de 95\% (IC=95\%), nível de significância de 5\% $(\alpha=0,05)$.

Os idosos foram convidados a participar da pesquisa individualmente, de acordo com a procura por atendimento na UBS. Foram explicados os objetivos e os aspectos éticos sobre a pesquisa. Após sanadas as eventuais dúvidas, as pessoas idosas e cuidadores assinaram o Termo de Consentimento Livre e Esclarecido (TCLE).

Delimitou-se como critérios de inclusão: ter idade maior ou igual a 60 anos de ambos os sexos; residir em domicílio cadastrado na UBS; apresentar capacidade cognitiva identificada pelo Miniexame do Estado Mental $^{12}$ de acordo com pontos de corte propostos segundo a escolaridade ${ }^{13}$ ou estar acompanhado de um cuidador familiar no momento da coleta de dados, quando não apresentar capacidade cognitiva para responder aos questionários.

Foram excluídas as pessoas idosas que residiam em instituições de longa permanência ou fisicamente incapazes de realizar os testes propostos, ser cadeirante ou apresentar amputação de membros inferiores e/ou superiores.

Três pesquisadores previamente treinados aplicaram o Miniexame do Estado Mental (MEEM), e posteriormente a coleta de dados foi realizada por meio de questionário sociodemográfico e clínico, escala de depressão e testes de avaliação da Fragilidade Física. Como medidas para a minimização dos riscos e proteção aos idosos participantes, os testes de força de preensão manual e velocidade da marcha foram 
aplicados por dois pesquisadores simultaneamente, para promover maior segurança e evitar episódio de queda do participante durante a realização do teste de velocidade da marcha.

A caracterização sociodemográfica e clínica foi composta pelas covariáveis: idade, sexo, escolaridade, estado civil, renda familiar morbidades existentes e/ou autorrelatadas. Utilizou-se a escala Center for Epidemiological Studies (CES-D) para o rastreio de sintomas depressivos. A Escala contém 20 itens sobre humor, sintomas somáticos, interações com os outros e funcionamento motor. As respostas são em Escala Likert, e o escore varia de 0 a 60 pontos. Pessoas idosas com a pontuação de 12 a 60 é indicativo da presença de $\mathrm{SD}^{14}$.

Para avaliação da fragilidade física consideraramse os cinco marcadores do fenótipo de Fried ${ }^{2}$, que classifica os indivíduos com três ou mais critérios como frágeis; pré-frágeis, com um ou dois critérios; e não frágeis os que não apresentam qualquer um dos seguintes componentes: força de preensão manual (FPM) reduzida, velocidade da marcha (VM) reduzida, fadiga/exaustão, perda de peso não intencional e redução do nível de atividade física.

A FPM foi aferida por meio de dinamômetro hidráulico Jamar $^{\circledR}$, considerando-se a média das três aferições realizados pela pessoa idosa, aqueles que compreenderam o quintil mais baixo de força foram considerados marcadores de fragilidade ${ }^{2}$. Para a avaliação da VM, a pessoa idosa foi orientada a caminhar, de maneira habitual, contabilizando o percurso dos 4,6 metros. Após o ajuste de sexo e mediana da altura, consideraram-se aqueles com quintil mais baixo, frágil para esse componente ${ }^{2}$.

A fadiga/exaustão foi identificada por autorrelato, conforme resposta do idoso aos itens 7 e 20 da Escala de depressão Center for Epidemiological Scale - Depression $(C E S-D)^{14}$, (A) sentiu que teve que fazer esforço para dar conta de suas tarefas de todos os dias; e (B) sentiu que não conseguiu levar adiante suas coisas. A perda de peso não intencional foi verificada pelo índice de massa corporal (IMC), calculado a partir de medidas antropométricas e associada ao autorrelato da pessoa idosa. Considerou-se a perda de peso corporal maior ou igual a 4,5 Kg nos últimos doze meses, de forma não intencional (sem dieta ou exercício) ${ }^{2}$.
A redução do nível de atividade física foi avaliada pelo Minnesota Leisure Activity Questionnaire, validado para pessoas idosas brasileiras ${ }^{15}$, o qual inclui perguntas quanto à frequência e tempo de atividades realizadas no último ano.

Os dados foram organizados e apresentado por estatística descritiva (frequência absoluta e relativa), inferencial (qui-quadrado de Pearson) com nível de significância estatístico de $p \leq 0,05)$. Foram ajustados modelos de regressão logística com diferente estrutura no preditor linear. Em um primeiro momento, foi realizado um modelo, individualmente, para cada marcador de fragilidade física e a condição de fragilidade física em relação aos SD. Em seguida, avaliou-se conjuntamente o efeito dos marcadores nos $\mathrm{SD}$, ajustando um único modelo com seleção das variáveis (backward) mediante Teste de Razão de Verossimilhança (TRV) ao nível de 5\%. Para os modelos reportou-se a estimativa e valor $p$ (teste de Wald), Razão de Prevalência (RP) com intervalo de confiança $95 \%$, bem como as medidas de acurácia, sensibilidade, especificidade, e os coeficientes Pseudo $\mathrm{R}^{2}$ de Mc Fadden, Cox e Snell, e Nagelkerke.

O estudo seguiu as recomendações contidas na Resolução no 466/2012 e na Resolução no 510/2016. Depois e foi encaminhado ao Comitê de Ética do Setor de Ciências da Saúde da Universidade Federal do Paraná, o qual recebeu parecer favorável sob o número 2.918.847.

\section{RESULTADOS}

Dos 389 participantes, foi identificada idade média de 70,45 $\pm 6,87$ anos (60-94). Houve predomínio do sexo feminino ( $\mathrm{n}=255 ; 65,6 \%)$, com baixa escolaridade ( $\mathrm{n}=138 ; 35,5 \%)$, casados $(\mathrm{n}=187 ; 48,1 \%)$, com renda familiar de 2-4 salários mínimos ( $\mathrm{n}=156$; 40,1\%). Quanto à condição de fragilidade,186 (47,8\%) idosos eram pré-frágeis, 169 (43,4\%) não frágeis e $34(8,8 \%)$ frágeis.

Os SD foram observados em 103 (26,5\%, IC95\%; $22,2 \%-31,2 \%$ ) das pessoas idosas. Dentre eles, e houve predomínio de pré-frágeis $(\mathrm{n}=63 ; 61,2 \%)$, seguido de não-frágeis $(\mathrm{n}=21 ; 20,4 \%)$ e frágeis $(\mathrm{n}=19$; 18,4\%) (Tabela 1). 
Em relação aos marcadores de fragilidade, foram associados aos SD a fadiga/exaustão, redução do nível de atividade física e perda de peso não intencional aos SD, conforme apresentado na Tabela 1 .

A condição de fragilidade também foi associada aos SD ( $p<0,001)$. O modelo de regressão logística da condição de fragilidade em relação aos SD indicou que a prevalência dos SD em pessoas idosas pré-frágeis foi 2,6 vezes quando comparado a pessoas idosas não frágeis $(p<0,001)$. Na condição frágil, a prevalência dos SD foi 3,4 vezes a das pessoas idosas não frágeis. Comparado aos pré-frágeis, as pessoas idosas com fragilidade tiveram ainda 1,85 vezes mais SD (Tabela 2).

Tabela 1. Distribuição de frequências absoluta e relativa e associação entre os marcadores, a condição de fragilidade e os SD das pessoas idosas. Curitiba, Paraná, Brasil, 2020.

\begin{tabular}{|c|c|c|c|c|}
\hline \multirow{2}{*}{ Marcadores de fragilidade } & \multirow{2}{*}{$\begin{array}{l}\text { Condição de } \\
\text { Fragilidade } \\
\text { n }(\%)\end{array}$} & \multicolumn{2}{|c|}{$\begin{array}{l}\text { Sintomas depressivos } \\
(\mathrm{n}=389)\end{array}$} & \multirow[t]{2}{*}{ Valor $p$} \\
\hline & & $\begin{array}{l}\operatorname{Sim} \\
\mathrm{n}=103\end{array}$ & $\begin{array}{l}\text { Não } \\
\mathrm{n}=286\end{array}$ & \\
\hline Força de Preensão Manual diminuída & & & & 0,076 \\
\hline $\operatorname{Sim}$ & & $28(27,2 \%)$ & $54(18,9 \%)$ & \\
\hline Não & & $75(72,8 \%)$ & $232(81,1 \%)$ & \\
\hline Velocidade da Marcha reduzida & & & & 0,491 \\
\hline $\operatorname{Sim}$ & & $10(9,7 \%)$ & $35(12,2 \%)$ & \\
\hline Não & & $93(90,3 \%)$ & $251(87,8 \%)$ & \\
\hline Fadiga/Exaustão & & & & $<0,001^{*}$ \\
\hline $\operatorname{Sim}$ & & $52(50,5 \%)$ & $14(4,9 \%)$ & \\
\hline Não & & $51(49,5 \%)$ & $272(95,1 \%)$ & \\
\hline Perda de peso não intencional & & & & $0,003^{*}$ \\
\hline Sim & & $32(31,1 \%)$ & $49(17,1 \%)$ & \\
\hline Não & & $71(68,9 \%)$ & $237(82,9 \%)$ & \\
\hline Redução do nível de atividade física & & & & $<0,001^{*}$ \\
\hline $\operatorname{Sim}$ & & $37(35,9 \%)$ & $45(15,7 \%)$ & \\
\hline Não & & $66(64,1 \%)$ & $241(84,3 \%)$ & \\
\hline Condição de Fragilidade & & & & $<0,001^{*}$ \\
\hline Não-frágil & $148(51,7 \%)$ & $21(20,4 \%)$ & $169(43,4 \%)$ & \\
\hline Pré-frágil & $123(43,0 \%)$ & $63(61,2 \%)$ & $186(47,8 \%)$ & \\
\hline Frágil & $15(5,2 \%)$ & $19(18,4 \%)$ & $34(8,7 \%)$ & \\
\hline
\end{tabular}

*Teste Qui-quadrado, $\mathrm{p} \leq 0,05$

Tabela 2. Modelo de regressão da condição de fragilidade em relação aos SD das pessoas idosas. Curitiba, Paraná, Brasil, 2020.

\begin{tabular}{|c|c|c|c|c|c|c|c|}
\hline \multirow[b]{2}{*}{ Condição de fragilidade } & \multirow[b]{2}{*}{ Estimativa } & \multirow[b]{2}{*}{$\mathrm{RP}$} & \multirow[b]{2}{*}{$\begin{array}{l}\text { IC p/ RP } \\
(95 \%)\end{array}$} & \multirow[b]{2}{*}{ Valor $p$} & \multicolumn{3}{|c|}{ Medidas de qualidade de ajuste } \\
\hline & & & & & $\begin{array}{l}\text { Coxsnell } \\
\mathrm{R}^{2}(\%)\end{array}$ & $\begin{array}{l}\text { Nagelkerke } \mathrm{R}^{2} \\
(\%)\end{array}$ & $\begin{array}{l}\text { McFadden } \mathrm{R}^{2} \\
(\%)\end{array}$ \\
\hline Pré-frágil/Não frágil & 1,283 & 2,610 & 1,707-3,991 & $<0,001$ & & & \\
\hline Frágil/Não frágil & 2,189 & 3,373 & $2,407-4,706$ & $<0,001$ & 9,310 & 13,586 & 8,453 \\
\hline Frágil/Pré-frágil & 0,905 & 1,853 & $1,186-2,896$ & 0,017 & & & \\
\hline
\end{tabular}

RP: Razão de Prevalência; IC: Intervalo de Confiança, $R^{2}$ Coxsnell, ${ }^{2}$ Nagelkerke, Pseudo $R^{2}$ Mcfadden. 
Na Tabela 3, evidencia-se a análise de regressão com os modelos ajustados de forma independente para cada marcador de fragilidade em relação aos sintomas depressivos. $\mathrm{Na}$ análise dos cinco componentes da fragilidade, três apresentaram valor de $p$ estatisticamente significativos: fadiga/exaustão $(p<0,001)$ perda de peso não intencional $(p<0,003)$ e redução da atividade física $(p<0,001)$. Desses, o modelo com a covariável fadiga/exaustão obteve melhores (e maiores) coeficientes das medidas de qualidade de ajuste.

O modelo de regressão final, após a seleção das variáveis, evidenciou que uma pessoa idosa com fadiga/exaustão apresentou 5,11 vezes maior prevalência de apresentar SD quando comparado a outra pessoa idosa que não apresenta esse marcador de fragilidade. O modelo ainda inclui o marcador de diminuição do nível de atividade física, indicando que uma pessoa idosa nessa condição apresentou 2,16 vezes maior prevalência de apresentar SD em relação àquelas sem redução de atividade física (Tabela 4).

Ao considerar as covariáveis idade, sexo, escolaridade, estado civil e renda familiar e os marcadores de fragilidade no modelo de regressão, somente a fadiga/exaustão e a redução do nível de atividade física permaneceram no modelo, evidenciando que os resultados independem das covariáveis sociodemográficas.

Tabela 3. Modelos de regressão para os SD das pessoas idosas ajustados de forma independente para cada marcador de fragilidade física e medidas de qualidade de ajuste. Curitiba, Paraná, Brasil, 2020.

\begin{tabular}{|c|c|c|c|c|c|c|c|}
\hline \multirow[b]{2}{*}{ Marcador de fragilidade } & \multirow[b]{2}{*}{ Estimativa } & \multirow[b]{2}{*}{$\mathrm{RP}$} & \multirow{2}{*}{$\begin{array}{l}\text { IC p/ RP } \\
(95 \%)\end{array}$} & \multirow[b]{2}{*}{ Valor $p$} & \multicolumn{3}{|c|}{ Medidas de qualidade de ajuste } \\
\hline & & & & & $\begin{array}{l}\text { Coxsnell } \\
\mathrm{R}^{2}(\%)\end{array}$ & $\begin{array}{l}\text { Nagelkerke } \\
\mathrm{R}^{2}(\%)\end{array}$ & $\begin{array}{l}\text { McFadden } \\
\mathrm{R}^{2}(\%)\end{array}$ \\
\hline $\begin{array}{l}\text { Diminuição da Força de } \\
\text { preensão manual }\end{array}$ & 0,472 & 1,397 & $0,975-2,001$ & 0,078 & 0,773 & 1,128 & 0,671 \\
\hline $\begin{array}{l}\text { Redução da Velocidade } \\
\text { da Marcha }\end{array}$ & $-0,259$ & 0,822 & $0,463-1,458$ & 0,492 & 0,125 & 0,183 & 0,108 \\
\hline Fadiga / Exaustão & 2,986 & 4,989 & $3,766-6,610$ & $<0,001$ & 22,611 & 32,996 & 22,174 \\
\hline $\begin{array}{l}\text { Perda de peso não } \\
\text { intencional }\end{array}$ & 0,779 & 1,713 & $1,222-2,403$ & 0,003 & 2,138 & 3,120 & 1,869 \\
\hline $\begin{array}{l}\text { Redução do nível de } \\
\text { atividade física }\end{array}$ & 1,099 & 2,098 & $1,523-2,891$ & $<0,001$ & 4,327 & 6,315 & 3,827 \\
\hline
\end{tabular}

RP: Razão de Prevalência; IC: Intervalo de Confiança, $\mathrm{R}^{2}$ Coxsnell, $\mathrm{R}^{2}$ Nagelkerke, Pseudo $\mathrm{R}^{2}$ Mcfadden. RP e IC(95\%) foram calculados a partir do preditor linear e estão na escala da Razão de Prevalência (RP).

Tabela 4. Variáveis preditoras do modelo preditivo final dos sintomas depressivos. Curitiba, Paraná, Brasil, 2020.

\begin{tabular}{|c|c|c|c|c|c|c|c|}
\hline \multirow[b]{2}{*}{ Marcador de fragilidade } & \multirow[b]{2}{*}{ Estimativa } & \multirow[b]{2}{*}{$\mathrm{RPa}$} & \multirow{2}{*}{$\begin{array}{l}\text { IC p/ RP } \\
(95 \%)\end{array}$} & \multirow[b]{2}{*}{ Valor $p$} & \multicolumn{3}{|c|}{ Medidas de qualidade de ajuste } \\
\hline & & & & & $\begin{array}{l}\text { Coxsnell } \\
\mathrm{R}^{2}(\%) \\
\end{array}$ & $\begin{array}{l}\text { Nagelkerke } \\
\mathrm{R}^{2}(\%)\end{array}$ & $\begin{array}{l}\text { McFadden } \\
\mathrm{R}^{2}(\%)\end{array}$ \\
\hline Fadiga/ Exaustão & 2,983 & 5,116 & $3,811-6.869$ & $<0,0001$ & & & \\
\hline $\begin{array}{l}\text { Redução do Nível de } \\
\text { atividade física }\end{array}$ & 1,092 & 2,160 & $1,450-3,218$ & $<0,0001$ & 24.986 & 36.462 & 24.870 \\
\hline
\end{tabular}

RPa: Razão de Prevalência ajustada; IC - Intervalo de Confiança, ${ }^{2}$ Coxsnell, $R^{2}$ Nagelkerke, Pseudo ${ }^{2}$ Mcfadden; RPa e IC( $\left.95 \%\right)$ foram calculados a partir do preditor linear e estão na escala da Razão de Prevalência (RP). 


\section{DISCUSSÃO}

O presente estudo investigou quais os marcadores de fragilidade física podem predizer a ocorrência de SD em pessoas idosas. As análises preditivas indicaram a maior chance das pessoas idosas que apresentam fadiga/exaustão e baixo nível de atividade física, desenvolverem SD. Além disso, a condição de fragilidade, obtida por meio desses e outros marcadores, explicam que quanto maior for o nível da condição de fragilidade, maior será a chance de desenvolverem os SD. Houve prevalência do marcador fadiga/ exaustão entre as pessoas idosas com SD, seguido da redução do nível de atividade física e da perda de peso não intencional.

A prevalência de SD entre as pessoas idosas foi superior à média nacional, estimada em uma revisão sistemática com metanálise realizada com pessoas idosas residentes na comunidade ${ }^{16}$. Estimativas inferiores foram identificadas na literatura nacional e internacional. Em Pelotas (RS), pesquisadores estimaram a prevalência de SD em 15,2\% $0^{17}, 14,2 \%$ em São Paulo (SP) ${ }^{18}, 9,8 \%$ na Austrália e 5,0\% nos $\mathrm{EUA}^{19}$. A variabilidade dos percentuais de SD entre as pessoas idosas em diferentes cidades brasileiras, devese aos diferentes métodos utilizados para classificação dos SD, instrumentos utilizados e às características das amostras ${ }^{16}$.

Observou-se o predomínio do sexo feminino na amostra de pessoas idosas com sintomas depressivos. Esse resultado foi consistente com outros estudos que mostraram as mulheres com maior risco de desenvolverem SD, comparado aos homens ${ }^{20,21}$.

Ainda que a maior prevalência no sexo feminino não seja universal, as mulheres experimentam mais a progressão acentuada dos SD ao longo do tempo ${ }^{22}$. Essa elevada frequência de SD entre as mulheres sugere a maior vulnerabilidade social delas em relação aos homens. Essa condição pode ser explicada pelas diferenças fisiológicas e hormonais, baixo nível de escolaridade, baixa renda e questões socioculturais ${ }^{23}$.

A proporção das pessoas idosas na condição de fragilidade foi analisada na amostra geral $(\mathrm{n}=389)$ e observou-se que o percentual de pré-fragilidade e fragilidade estavam elevados entre as pessoas idosas com SD. Ambas as condições (frágeis e pré-frágeis) foram associadas à sintomatologia depressiva.

As associações transversais entre pré-fragilidade, fragilidade e sintomatologia depressiva verificadas neste estudo, também foram observadas em pesquisa brasileira com 2.042 pessoas idosas comunitárias que analisou relações entre a presença de depressão e de SD específicos e as condições de pré-fragilidade e fragilidade. Os resultados também indicaram a associação entre SD e a condição de fragilidade $(p<0,001)^{24}$.

Os mecanismos das associações entre a fragilidade e os transtornos depressivos ainda são pouco claros e dificultados pela sobreposição sintomas. Pessoas idosas frágeis podem desenvolver mais facilmente os SD devido ao comprometimento da funcionalidade, baixa atividade física e atividades sociais. Em nível molecular, pessoas idosas frágeis podem ter aumentado níveis de inflamação de baixo grau, como aumento citocinas de interleucina-6, proteína $C$ reativa ou fator de necrose tumoral- $\alpha$, que podem agir como como moderados do risco do início dos $\mathrm{SD}^{11,25}$.

Os marcadores fadiga/exaustão, redução do nível de atividade física apresentaram forte associação com SD. As mudanças no comportamento motor, como fraqueza associada à depressão, podem contribuir para o baixo nível de atividade física. Estudo realizado no Japão, com 3.191 idosos da comunidade, demonstrou que a atividade física foi associada a um risco reduzido de SD independente da frequência e duração da atividade ${ }^{26}$. A atividade física realizada de forma individualizada ou grupo, envolve sentimentos de prazer, autoestima, diversão, facilita a mudança de foco da dor e da solidão, alivia a tensão. Dessa forma, as pessoas idosas devem ser encorajadas ao envolvimento de atividades físicas em seu cotidiano.

Considera-se que os sintomas depressivos e a fragilidade física têm impacto negativo no funcionamento físico, psicossocial, perda da independência e autonomia da pessoa idosa. A inclusão de práticas que visam a minimizar ou evitar tais condições de saúde se torna necessária nos diferentes níveis de atenção, especialmente na Atenção Primária à Saúde. 
No estudo observou-se que quanto maior a condição de fragilidade, maior a chance de a pessoa idosa apresentar SD. Esses resultados corroboram ao estudo transversal realizado em Singapura, com uma amostra de 721 pessoas idosas comunitárias com idade igual ou maior de 60 anos. O estudo identificou prevalência de fragilidade em $24,5 \%$ das pessoas idosas e a associação independente entre o nível de fragilidade e SD. Verificou-se que com o aumento no nível de gravidade da fragilidade, os idosos relataram escores de SD substancialmente mais altos ${ }^{25}$.

No presente estudo, a condição de fragilidade foi identificada como um preditor dos SD em pessoas idosas. Dados semelhantes foram apontados em estudo de revisão sistemática que identificou o nível de fragilidade como preditor longitudinal de sintomatologia depressiva. O estudo observou que um em cada dez pessoas idosas apresentou SD, e identificou elevado percentual de pessoas idosas com SD e na condição de fragilidade ${ }^{27}$. Nota-se a importância e a necessidade de se observar que a presença de SD afeta comportamentos e níveis de atividade física da pessoa idosa com consequente redução da participação social, risco de depressão e aumento da fragilidade.

A presença do marcador redução do nível de atividade física no modelo, confere à pessoa idosa a prevalência 2,16 vezes maior de apresentar SD, comparados àqueles sem o respectivo marcador. Já evidências apontam a atividade física como fator protetor de $\mathrm{SD}^{28,29}$. Os benefícios da atividade física para a manutenção da saúde nas pessoas idosas têm sido consistentes com resultados ou associações positivas no humor, autoestima e menor ocorrência de SD.

Quanto à associação entre fadiga/exaustão e SD também foi identificada em outros estudos na literatura, sendo considerada como um importante indicador de declínio relacionado ao envelhecimento e fortemente associada aos eventos negativos à saúde $\mathrm{e}^{18,30}$.

Os modelos preditivos analisados confirmam a importância da avaliação da fadiga/exaustão e do baixo nível de atividade física para a prevenção do desenvolvimento dos SD nas pessoas idosas comunitárias. A abordagem na prática clínica com foco na fadiga e exaustão e no baixo nível de atividade física são fundamentais para o rastreio dos idosos com risco de apresentar SD tanto no contexto da Atenção Primária à Saúde, quanto em redes ambulatoriais que atendem usuários com esse segmento etário.

A fadiga/exaustão por autorrelato deve ser prioridade na prática clínica e nos cuidados gerontológicos direcionando maior atenção para essa condição, frente aos desfechos adversos à saúde da pessoa idosa, como a inatividade física, as quedas, a hospitalização e a menor qualidade de vida ${ }^{31}$.

Devido à escassa reserva energética, é comum o esgotamento que, por vezes, é admitido como um sintoma da velhice, no entanto pode advir de SD e dos primeiros sinais de mau prognóstico da fragilidade ${ }^{1,32}$. São condições mensuráveis e modificáveis se identificadas precocemente, o que contribui para a prevenção e minimização de desfechos como os SD e a fragilidade na população idosa.

A causalidade da presença de SD nas pessoas idosas avaliadas nesta pesquisa são desconhecidas, mas a forte associação aos marcadores de fragilidade que atuam em uma cascata incapacitante, com impacto na autonomia da pessoa idosa e o potencial desenvolvimento de SD sugere a importância da avaliação da fragilidade física e das causas da fadiga/ exaustão autorreferidas.

Como limitações do estudo, ressalta-se que os dados foram obtidos em um estudo do tipo transversal. Desse modo, inferências sobre causalidade da relação entre as variáveis preditoras e os SD devem ser feitas com cautela, uma vez que se trata de uma pesquisa com resultados de uma população local na região de Curitiba, Paraná. Outra limitação se refere aos instrumentos com questões de autorrelato para avaliação dos SD, o que pode gerar vieses devido à necessidade de a pessoa idosa reportar sentimentos e/ou memórias. O instrumento Minnesota Leisure Time Activities para avaliação do nível de atividade física contém atividades não compatíveis com a realidade da população brasileira, o que pode impactar na mensuração do gasto energético dos idosos. 


\section{CONCLUSÃO}

A condição e os marcadores de fragilidade fadiga/ exaustão e redução do nível de atividade física foram preditores de sintomas depressivos (SD) em pessoas idosas. A pré-fragilidade e fragilidade foi superior em pessoas idosas com SD na amostra estudada.

A pré-fragilidade se mostrou uma condição prevalente entre as pessoas idosas em que sintomas de fadiga e escassez de energia e de sensibilidade interpessoal somam-se a um rebaixamento em afetos positivos. Uma vez identificada a pessoa idosa frágil e pré-frágil, é importante considerar a forte relação entre essas condições.

Os marcadores de fragilidade fadiga/exaustão e redução do nível de atividade física demonstraram-se preditores de SD na pessoa idosa, sendo o primeiro, aquele com maior poder preditivo.

Os SD podem apresentar rastreio prejudicado devido à subjetividade dos sintomas relatados. $\mathrm{O}$ rastreio da fragilidade física, por sua vez, é realizado com enfoque na dimensão física da pessoa idosa, que facilita o diagnóstico e direciona o cuidado em saúde. Nesse contexto, destaca-se a importância da implementação do rastreio da fragilidade física na atenção primária à saúde.

Esses resultados favorecem a prática Geriátrica e Gerontológica, uma vez que ao avaliar as causas de queixas comuns relacionadas à fadiga e à redução das atividades da vida diária, o profissional identifica alterações precocemente, e possibilita condutas assertivas para pessoas idosas-frágeis e pré-frágeis com risco aumentado para desenvolvimento de sintomatologia depressiva. As ações podem ser direcionadas ao estímulo da prática de exercícios físicos e por meio da implementação de ações para a melhoria da gestão da fragilidade, que facultam a prevenção de SD. Desse modo, considerando a relação modificável que há nelas, atua-se na prevenção ou retardo dessas condições em pessoas idosas.

Editado por: Marquiony Marques dos Santos

\section{REFERÊNCIAS}

1. Dent E, Morley JE, Cruz-Jentoft AJ, Woodhouse L, Rodrígues-Mañas L, Fried LP, et al. Physical Frailty: ICFSR International Clinical Practice Guidelines for Identification and Management. J Nutr Health Aging . 2019;23(9):771-87.

2. Fried LP, Tangen CM, Walston J, Newman AB, Hirsch C, Gottdiener J, et al. Frailty in older adults: evidence for a phenotype. J Gerontol Ser A Biol Sci Med Sci. 2001;56(3):146-56.

3. O’Caoimh R, Galluzzo L, Rodríguez-Laso A, van der Heyden J, Ranhoff AH, Lamprini-Koula M, et al. Prevalence of frailty at population level in European ADVANTAGE Joint Action Member States: a systematic review and meta-analysis. Ann Ist Super Sanita. 2018;54(3):226-38.

4. Coelho-Junior HJ, Marzetti E, Picca A, Calvani R, Cesari M, Uchida MC. Prevalence of prefrailty and frailty in South America: a systematic review of observational studies. J Frailty Aging. 2020;9(4):197-213.

5. Cesari M, Pérez-Zepeda MU, Marzetti E. Frailty and multimorbidity: different ways of thinking about geriatrics. J Am Med Dir Assoc. 2017;18(4):361-64.
6. Soysal P, Veronese N, Thompson T, Kahl KG, Fernandes BS, Prina AM, et al. Relationship between depression and frailty in older adults: a systematic review and meta-analysis. Ageing Res Rev. 2017;36:78-87.

7. Lian Y, Yang L, Gao M, Jia CX. Relationship of frailty markers and socioeconomic status to incidence of depressive symptoms in a community cohort. J Am Med Dir Assoc. 2021;22(3):570-6.

8. Cruz DT, Vieira MT, Bastos RR, Leite ICG. Factors associated with frailty in a community-dwelling population of older adults. Rev Saúde Pública. 2017;51:1-10.

9. Prina M, Stubbs B, Veronese N, Guerra M, Kralj C, Rodriguez J, et al. Depression and incidence of frailty in older people from Six Latin American Countries. Am J Geriatr Psychiatry. 2019;27(10):1072-79.

10. Pérez LM, Castellano-Tejedor C, Cesari M, SotoBagaria L, Ars J, Zambom-Ferraresi F, et al. Depressive symptoms, fatigue and social relationships influenced physical activity in frail older communitydwellers during the Spanish Lockdown due to the COVID-19 Pandemic. Int J Environ Res Public Health. 2021;19;18(2):1-10. 
11. Chu XF, Zhang N, Shi GP, Wang Y, Wang ZD, Guo $\mathrm{JH}$. Frailty and incident depressive symptoms in a Chinese sample: the Rugao Longevity and Ageing Study. Psychogeriatrics. 2020;20(5):691-8.

12. Folstein MF, Folstein SE, Mchugh PR. Mini-Mental State: a practical method for grading the cognitive status of patients for the clinician. J Psychiatr Res [Internet]. 1975;12(3):189-98.

13. Bertolucci PHF, Brucki SMD, Campacci SR, Juliano Y. Mini-exame do estado mental em uma população geral: impacto da escolaridade. Arq Neuropsiquiatr. 1994;52(1):1-7.

14. Batistoni SST, Neri AL, Cupertino APFB. Validade da escala de depressão do Center for Epidemiological Studies entre idosos brasileiros. Rev Saúde Pública. 2007;41(4):598-605.

15. Lustosa LP, Pereira DS, Dias RC, Brito RR, Parentoni AN, Pereira LSM. Tradução e adaptação transcultural do Minnesota Leisure Time Activities Questionnaire em idosos. Geriatr Gerontol [Internet]. 2011;5(2):5765. Disponível em: https://cdn.publisher.gn1.link/ ggaging.com/pdf/v5n2a03.pdf .

16. Meneguci J, Meneguci CAG, Moreira MM, Pereira KR, Tribess S, Sasaki JE, et al. Prevalência de sintomatologia depressiva em idosos brasileiros: uma revisão sistemática com metanálise. J Bras Psiquiatr. 2019;68(4):221-30.

17. Hellwig N, Munhoz TN, Tomasi E. Sintomas depressivos em idosos: estudo transversal de base populacional. Ciênc Saúde Colet [Internet]. 2016;21(11):3575-84. Disponível em: https://doi. org/10.1590/1413-812320152111.19552015 .

18. Mendes-Chiloff CL, Lima MCP, Torres AR, Santos JLF, Duarte YO, Lebrão ML, et al. Depressive symptoms among the elderly in São Paulo city, Brazil: prevalence and associated factors (SABE Study). Rev Bras Epidemiol [Internet]. 2019; 21(Suppl 02):e180014. Disponível em : https://doi.org/10.1590/1980549720180014.supl.2

19. Mohebbi M, Agustini B, Woods RL, McNeil JJ, Nelson MR, Shah RC, et al. Prevalence of depressive symptoms and its associated factors among healthy community-dwelling older adults living in Australia and the United States. Int J Geriatr Psychiatry. 2019;34(8):1208-1216.

20. Zhong BL, Xu YM, Xie WX, Liu XJ, Huang ZW. Depressive Symptoms in Elderly Chinese Primary Care Patients: Prevalence and Sociodemographic and Clinical Correlates. J Geriatr Psychiatry Neurol. 2019;32(6):312-8.
21. Conde-Sala JL, Garre-Olmo J, Calvó-Perxas L, Turró-Garriga O, Vilalta-Franch J. Course of depressive symptoms and associated factors in people aged 65+ in Europe: A two-year follow-up. J Affect Disord. 2019;245:440-50.

22. Wang R, Bishwajit G, Zhou Y, Wu X, Feng D, Tang $\mathrm{S}$, et al. Intensity, frequency, duration, and volume of physical activity and its association with risk of depression in middle- and older-aged Chinese: Evidence from the China Health and Retirement Longitudinal Study, 2015. PLoS ONE [Internet]. 2019;14(8):e0221430. Disponível em: https://doi. org/10.1371/journal.pone.0221430 .

23. Gonçalves AMC, Teixeira MTB, Gama JRA, Lopes CS, Silva GA, Gamarra CJ, et al. Prevalence of depression and associated factors in women covered by Family Health Strategy. J Bras Psiquiatr [Internet]. 2018;67(2):101-9. Disponível em: https://doi. org/10.1590/0047-2085000000192 .

24. Nascimento PPP, Batistoni SST, Neri AL. Frailty and depressive symptoms in older adults: data from the FIBRA study - UNICAMP. Psicol. Reflex. Crit [Internet]. 2016;29(4):778-92. Disponível em: https:// doi.org/10.1186/s41155-016-0033-9 .

25. Ge L, Yap CW, Heng BH. Prevalence of frailty and its association with depressive symptoms among older adults in Singapore. Aging Ment Health. 2019;23(3):319-24..

26. Jung S, Lee S, Lee S, Bae S, Imaoka M, Harada K, et al. Relationship between physical activity levels and depressive symptoms in community-dwelling older Japanese adults. Geriatr Gerontol Int. 2018;18(3):421-7.

27. Vaughan L, Corbin AL, Goveas JS. Depression and frailty in later life: a systematic review. Clin Interv Aging. 2015;10:1947-58.

28. Kim SY, Park JH, Lee MY, Oh KS, Shin DW, Shin YC. Physical activity and the prevention of depression: A cohort study. Gen Hosp Psychiatry. 2019;60:90-7.

29. Kandola A, Ashdown-Franks G, Hendrikse J, Sabiston CM, Stubbs B. Physical activity, and depression: Towards understanding the antidepressant mechanisms of physical activity. Neurosci Biobehav Rev. 2019;107:525-39.

30. Matoso LBBMM, Boing L, Korpalski T, Dias M, Moratelli J, Fausto DY, et al. Relationship of fatigue with depressive symptoms and level of physical activity in women with breast cancer diagnosis. Rev Bras Cineantropom Desempenho Hum [Internet]. 2020;22(3):e59189. Disponível em: https://doi. org/10.1590/1980-0037.2020v22e59189 . 
31. Silva SLAD, Neri AL, Ferrioli E, Lourenço RA, Dias RC. Fenótipo de fragilidade: influência de cada item na determinação da fragilidade em idosos comunitários - Rede Fibra. Ciênc Saúde Colet [Internet]. 2016;21(11):3483-92. Disponível em: https://doi.org/10.1590/1413-812320152111.23292015 .
32. Pao YC, Chen CY, Chang CI, Chen CY, Tsai JS. Self-reported exhaustion, physical activity, and grip strength predict frailty transitions in older outpatients with chronic diseases. Medicine [Internet]. 2018;97(23):e10933. Disponível em: https://doi. org/10.1097/md.0000000000010933 . 